\title{
Antiplatelet drug ticagrelor delays gastric ulcer healing in rats
}

\author{
JING-JING LI ${ }^{1}$, XIN-YING WU ${ }^{2}$, JING-LOU CHEN ${ }^{1}$, GUAN-RONG CHEN ${ }^{1}$, \\ JUN XU ${ }^{1}, \mathrm{YE} \mathrm{GU}^{2}$ and HONG-PING SONG ${ }^{1}$ \\ Departments of ${ }^{1}$ Pharmacy and ${ }^{2}$ Cardiology, Puai Hospital, Tongji Medical College, \\ Huazhong University of Science and Technology, Wuhan, Hubei 430000, P.R. China
}

Received April 6, 2016; Accepted March 24, 2017

DOI: $10.3892 /$ etm.2017.4955

\begin{abstract}
Adenosine diphosphate $\mathrm{P} 2 \mathrm{Y}_{12}$ receptor antagonist clopidogrel is not sufficiently safe for the gastric mucosa in patients with high risk of peptic ulcer, since it may impair healing of gastric erosions. However, the safety of the novel $\mathrm{P} 2 \mathrm{Y}_{12}$ receptor antagonist ticagrelor in the gastric mucosa has not been elucidated to date. The present study aimed to examine whether ticagrelor delays gastric ulcer healing and to elucidate the involved mechanisms. Gastric kissing ulcers were produced in rats by luminal application of acetic acid solution, and ticagrelor was administered at dose of 10 or $20 \mathrm{mg} / \mathrm{kg} /$ day orally for 7 days. On day 8 after ulcer induction, the ulcer size, mucosal epithelial cell proliferation of the ulcer margin, expression levels of epidermal growth factor (EGF) and vascular endothelial growth factor (VEGF), and signal transduction pathways for cell proliferation and angiogenesis were measured and compared between the ticagrelor-treated and untreated model groups. The results revealed that the ulcer size was significantly greater in the ticagrelor-treated group compared with the model group, while the mucosal epithelial cell proliferation of the ulcer margin was significantly decreased in the ticagrelor-treated group. In addition, ticagrelor significantly decreased the ulcer-stimulated expression levels of EGF, VEGF, phosphorylated extracellular signal-regulated kinase (ERK), phosphorylated P38 mitogen-activated protein kinase and nuclear factor- $\kappa$ B P65 at the ulcer margin $(\mathrm{P}<0.05)$. These findings suggested that ticagrelor delayed gastric ulcer healing. Furthermore, the possible mechanisms underlying the effect of ticagrelor were associated with its functions of
\end{abstract}

Correspondence to: Dr Xin-Ying Wu, Department of Cardiology, Puai Hospital, Tongji Medical College, Huazhong University of Science and Technology, 473 Hanzheng Road, Wuhan, Hubei 430000, P.R. China

E-mail: wuxingying81@126.com

Professor Hong-Ping Song, Department of Pharmacy, Puai Hospital, Tongji Medical College, Huazhong University of Science and Technology, 473 Hanzheng Road, Wuhan, Hubei 430000, P.R. China E-mail: 42441612@qq.com

Key words: cell proliferation, angiogenesis, $\mathrm{P} 2 \mathrm{Y}_{12}$ receptor antagonist, ulcer healing attenuating the expression levels of VEGF and EGF, as well as suppressing the phosphorylation activation of ERK1/2, P38 and nuclear factor- $\kappa$ B P65. Finally, the gastric epithelial cell proliferation and angiogenesis were also inhibited.

\section{Introduction}

In patients who have acute coronary syndromes with or without ST-segment elevation, current clinical practice guidelines recommend dual antiplatelet treatment with aspirin and adenosine diphosphate (ADP)-receptor $\mathrm{P}_{2} \mathrm{Y}_{12}$ antagonists, including clopidogrel or ticagrelor $(1,2)$. However, these antiplatelet agents have recognizable risks, in particular, gastrointestinal complications, including ulceration and related bleeding (3). The absolute increased risk per year of upper gastrointestinal events with aspirin is high (0.12\%) when compared with placebo (4), and aspirin is associated with a 2- to 4-fold increase in gastrointestinal bleeding (5). Combined aspirin and clopidogrel is associated with significantly increased risk of gastrointestinal bleeding when compared with aspirin alone [risk ratio (RR), 1.86; 95\% confidence interval (CI), 1.49-2.13] (4). Clopidogrel may not be a primary cause of gastroduodenal ulcers, and may impair healing of gastric erosions or small ulcerations that develop as a result of other medications or Helicobacter pylori infection (6).

Ticagrelor is a novel oral, reversible, direct-acting inhibitor of the ADP receptor $\mathrm{P} 2 \mathrm{Y}_{12}$ with a more rapid onset and pronounced platelet inhibition in comparison with clopidogrel, which is another $\mathrm{P}_{2} \mathrm{Y}_{12}$ receptor (7). Patients with acute coronary syndrome treated with ticagrelor present reduced mortality rates from vascular causes, myocardial infarction or stroke, as opposed to those receiving clopidogrel treatment (7). Therefore, the American College of Cardiology/American Heart Association has suggested that ticagrelor should be preferred to clopidogrel for patients treated with an early invasive or ischemia-guided strategy (1). In addition, the European Society of Cardiology recommended clopidogrel in acute coronary syndrome patients who cannot receive ticagrelor (2).

It is well known that clopidogrel is not sufficiently safe for the gastric mucosa in patients with a high risk of peptic ulcer, since its anti-angiogenic effects may impair healing of gastric erosions. Ticagrelor has also been found to increase the risk of gastrointestinal/anal bleeding $(\mathrm{R}=1.23$; $95 \%$ confidence interval, 0.93-1.64) in a PLATO trail (8). However, to date, the 
potential impairment in healing of gastric erosions as a result of ticagrelor treatment have not been elucidated. The present study aimed to investigate whether ticagrelor delays gastric ulcer healing and to elucidate the involved mechanisms in a rat model of gastric kissing ulcers.

\section{Materials and methods}

Animals. A total of 32 male Sprague-Dawley rats (6 weeks old) weighing 180-200 g were obtained from Hubei Provincial Center for Disease Control and Prevention, and kept in a room with controlled temperature $\left(22 \pm 3^{\circ} \mathrm{C}\right)$ and humidity $(50 \pm 10 \%)$, and $12 \mathrm{~h}$ dark/light cycles. The rats were fed standard laboratory chow and tap water ad libitum, and acclimated 7 days before they were used for the study. All experimental procedures were performed under the Guidelines for the Care and Use of Laboratory Animals (Science and Technology Department, Hubei, China). The study protocol was approved by the Institutional Animal Care and Use Committee of Wuhan Puai Hospital (approval no. IACUC-105; Wuhan, China).

Induction of gastric kissing ulcers. Following acclimatization to the laboratory conditions for 7 days, experimental gastric ulcers were induced in the rats (9). Briefly, the rats were anesthetized with thioethamyl (35 mg/kg; Beijing Propbs Bio Co., Ltd., Beijing, China) by intraperitoneal injection. Next, the abdomen was incised and the stomach was exposed. The anterior and posterior stomach walls were clamped together with forceps with a round ring (inside diameter, $7 \mathrm{~mm}$ ). A $60 \%(\mathrm{v} / \mathrm{v})$ acetic acid solution $(0.2 \mathrm{ml})$ was injected into the clamped lumen with a needle through the forestomach. After $45 \mathrm{sec}$, the acid was removed and the abdomen was closed (9). The animals were fed normally thereafter.

Drug treatment and measurement of gastric ulcer. One day after gastric ulcer induction, the animals were divided into three groups ( $n=8$ each) as follows: Model group, low dose ticagrelor group and high dose ticagrelor group. A further 8 rats, which underwent a sham surgery with similar surgical procedure, but with instillation of distilled water instead of acetic acid, were set as the sham group. The low and high dose ticagrelor groups were orally treated with 10 or $20 \mathrm{mg} / \mathrm{kg} /$ day ticagrelor (AstraZeneca AB, Södertälje, Sweden) for 7 consecutive days, respectively. Ticagrelor was dissolved in physiological saline with $0.5 \%$ sodium carboxymethyl cellulose (CMC). The sham and model groups received $0.5 \% \mathrm{CMC}$ in physiological saline without ticagrelor. The dosage of medicinal compounds was established according to previous study (10).

On day 8, all experimental animals were sacrificed. Each stomach was then opened along the greater curvature, and the areas $\left(\mathrm{mm}^{2}\right)$ of the ulcers were determined under a dissecting microscope (magnification, x10; Olympus Optical, Tokyo, Japan) with a square grid. The ulcer area was measured in a blinded manner.

Histological and immunohistochemical analysis. One ulcer part (1/3 of the ulcer) was excised, fixed with $4 \%$ paraformaldehyde, embedded in paraffin and serially sectioned $(\sim 6 \times 2 \mathrm{~mm})$ for histological and immunohistochemical examination. Gastric sections were stained with hematoxylin and eosin (H\&E), and histological analysis was conducted using an optical microscope (BX51; Olympus, Tokyo, Japan) at a magnification of $\mathrm{x} 40$. Additionally, immunohistochemical analysis was performed using deparaffinized ulcer sections. The sections were immersed in freshly prepared $2 \% \mathrm{H}_{2} \mathrm{O}_{2}$ at $37^{\circ} \mathrm{C}$ for $10 \mathrm{~min}$ and blocked with $5 \%$ goat serum for $10 \mathrm{~min}$. Subsequently, primary antibodies against vascular endothelial growth factor (VEGF, ab53465, dilution 1:200; Abcam, Cambridge, UK), epidermal growth factor (EGF, ab77851, dilution 1:200; Abcam) or proliferation cell nuclear antigen (PCNA, ab18197, dilution 1:500; Abcam) were added and incubated at $37^{\circ} \mathrm{C}$ for $1 \mathrm{~h}$. After washing with phosphate-buffered saline, the sections were treated with the secondary antibody conjugated with horseradish peroxidase at $37^{\circ} \mathrm{C}$ for 10 min according to the manufacturer's instructions of the kit (K500711; Dako; Agilent Technologies, Inc., Santa Clara, CA, USA). Sections were then immersed in diaminobenzidine for $3 \mathrm{~min}$. Following staining with hematoxylin for $1 \mathrm{~min}$ at room temperature, the sections were washed with $\mathrm{H}_{2} \mathrm{O}$, soaked in $1 \%$ hydrochloric acid for $3 \mathrm{sec}$ and dehydrated using ethanol. A negative control group was obtained by omitting the primary antibody and the other operations were the same. Stained areas of the sections were visualized using an optical microscope at magnification of $\mathrm{x} 100$.

Biochemical analysis. The remainder part of the tissue $(\sim 1 / 3$ of the ulcer) was used to prepare $10 \%$ tissue homogenate by addition of ice-cold physiological saline for the measurement of EGF and VEGF levels using EGF (EK0954) and VEGF (EK0540) ELISA commercial kits (Boster Biological Technology, Ltd., Wuhan, China) according to the manufacturer's instructions.

Western blot analysis. Gastric ulcer tissues were homogenized and centrifuged at $4^{\circ} \mathrm{C}$ for $10 \mathrm{~min}(9055.8 \mathrm{x} \mathrm{g})$ to obtain the supernatants. Protein concentration was measured using bicinchoninic acid protein assay kit (P0010; Beyotime Institute of Biotechnology, Shenzhen, China), and then $20 \mu$ proteins were separated by $10 \%$ SDS-PAGE and transferred to polyvinylidene fluoride membranes (EMD Millipore, Billerica, MA, USA). Transferred membranes were blocked for $1 \mathrm{~h}$ at room temperature with $5 \%$ non-fat milk in Tris-buffered saline containing $0.1 \%$ Tween-20. These membranes were then probed with rabbit primary antibodies against extracellular signal-regulated kinase (ERK1/2; 4695) and its phosphorylated form (P-ERK; 4370), P38 mitogen-activated protein kinase (MAPK; 8690) and its phosphorylated form (P-P38) (4511), nuclear factor- $\kappa \mathrm{B}(\mathrm{NF}-\kappa \mathrm{B})$ p65 (8242, all 1:1,000; Cell Signaling Technology, Inc., Danvers, MA, USA) and $\beta$-actin (20536-1-AP, 1:10,000; Proteintech Group, Inc., Wuhan, China) overnight at $4^{\circ} \mathrm{C}$. Subsequently, the membranes were incubated for $1 \mathrm{~h}$ with secondary antibodies conjugated with horseradish peroxidase (SA00001-2, 1:5,000; Proteintech Group, Inc.). Immunoblots were developed on films using the enhanced chemiluminescence technique (SuperSignal West Pico; Thermo Fisher Scientific, Inc., Waltham, MA, USA). Quantification of bands was determined by densitometric analysis using Bio-Rad Quantity One 4.52 (Bio-Rad Laboratories, Inc., Hercules, CA, USA). 

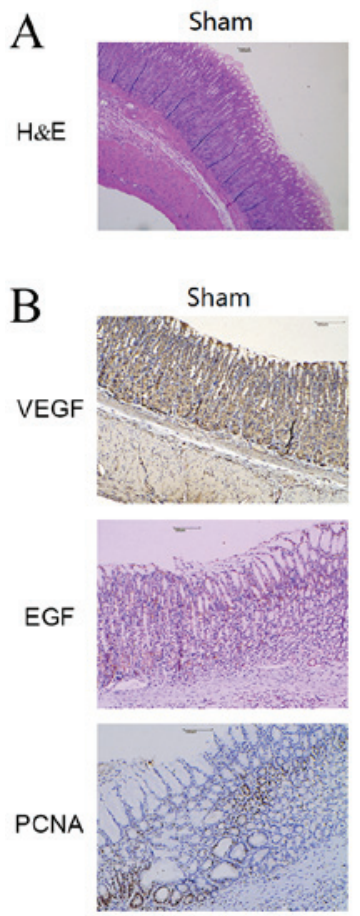
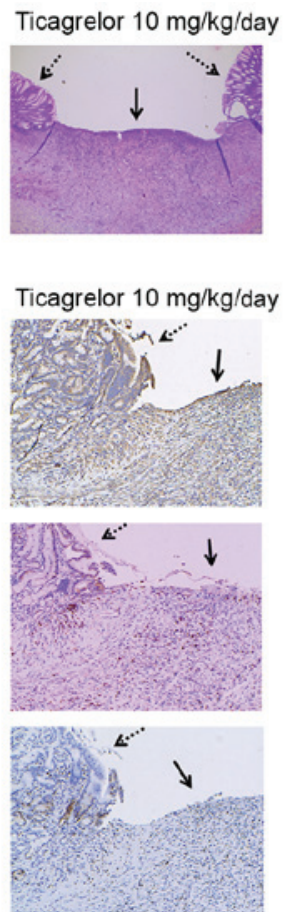
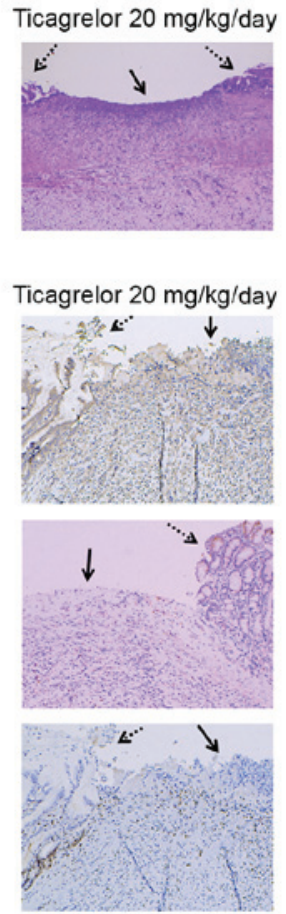

Figure 1. Histological and immunohistochemical analysis of gastric mucosal tissues of rats in the various groups. The filled arrow indicated the ulcer area internal. The dashed arrow indicated the ulcer edge. (A) Hematoxylin and eosin staining (magnification, x40), and (B) immunoreactivity for VEGF, EGF and PCNA antibodies (magnification, x100) are shown. VEGF, vascular endothelial growth factor; EGF, epidermal growth factor; PCNA, proliferation cell nuclear antigen.

Statistical analysis. Results are expressed as the mean \pm standard deviation. SPSS version 11.5 (IBM Corp., Armonk, NY, USA) was used to conduct analysis. The data were analyzed by one-way analysis of variance followed by Tukey's multiple comparison. $\mathrm{P}<0.05$ was considered to indicate a statistically significant difference.

\section{Results}

Ticagrelor effect on gastric ulcer healing. Delayed rat gastric ulcer healing on day 8 after ulcer induction was observed in the ticagrelor-treated groups when compared with the model group. Oral administration of ticagrelor (10 or $20 \mathrm{mg} / \mathrm{kg} / \mathrm{day}$ ) significantly increased the area of chronic ulcer $(26 \pm 6$ and $27 \pm 6 \mathrm{~mm}^{2}$, respectively) compared with the model group $\left(20 \pm 5 \mathrm{~mm}^{2}, \mathrm{P}<0.05\right)$. However, there was no significant difference between the low and high dose ticagrelor groups $(\mathrm{P}>0.05)$.

Histological analysis. Histological analysis by H\&E staining was performed to investigate the absence (ulcer area internal; filled arrow in Fig. 1A) or presence of the epithelial layer (ulcer edge; dashed arrow in Fig. 1A). In the model group, superficial mucosal necrosis was observed, and the structures of the glands, muscularis mucosa, submucosa and muscular were damaged. In addition, moderate amount of inflammatory cells infiltrated the basal layer of the ulcers, and a thin layer of granulation tissues with angiogenesis at the ulcer base was found. In the low and high dose ticagrelor groups, the size of the ulcer and the length of ruptured muscularis mucosa were larger when compared with the model group. Increased mucosal necrosis and inflammatory cell infiltration were also observed in the ticagrelor groups compared with model group.
Immunohistochemical analysis for VEGF, EGF and PCNA. As shown in Fig. 1B via semi-quantitative analysis using Image-Pro Plus 6.0, VEGF, EGF and PCNA were rarely expressed in sham group. However, cells in the ulcer edges and ulcer beds were strongly stained by the anti-VEGF antibody in the model group, while ticagrelor treatment decreased the expression of VEGF. Upon staining with anti-EGF antibody, only a small number of EGF immunopositive cells were observed in the bottom of the oxyntic glands in the gastric tissues of the sham group. Ulceration led to increased expression of EGF in the damaged epithelial portion, as well in the foveolar and glandular parts in the model group. Ticagrelor treatment decreased the number of EGF-positive cells, revealing decreased EGF expression, as compared with the sham group (Fig. 1A).

A moderate number of PCNA-positive nuclei in the layer adjacent to the ulcer were identified in the model group. The ticagrelor-treated groups demonstrated a lower percentage of labeled nuclei, suggesting that ticagrelor treatment delayed cell proliferation in the area of gastric mucosal healing.

Effect of ticagrelor on VEGF and EGF level at the ulcer margin. There were significant increases in the mucosal VEGF and EGF levels at the ulcer margin when compared with those of the sham mucosa, as determined by ELISA. By contrast, ticagrelor treatment dose-independently decreased mucosal VEGF and EGF levels at the ulcer margin when compared with those of the model group (Fig. 2).

Effect of ticagrelor on protein expression of ERK, P38 and $P 65$ at the gastric ulcer margin. Ulcer induction markedly increased the protein expression levels of P-ERK, P-P38 

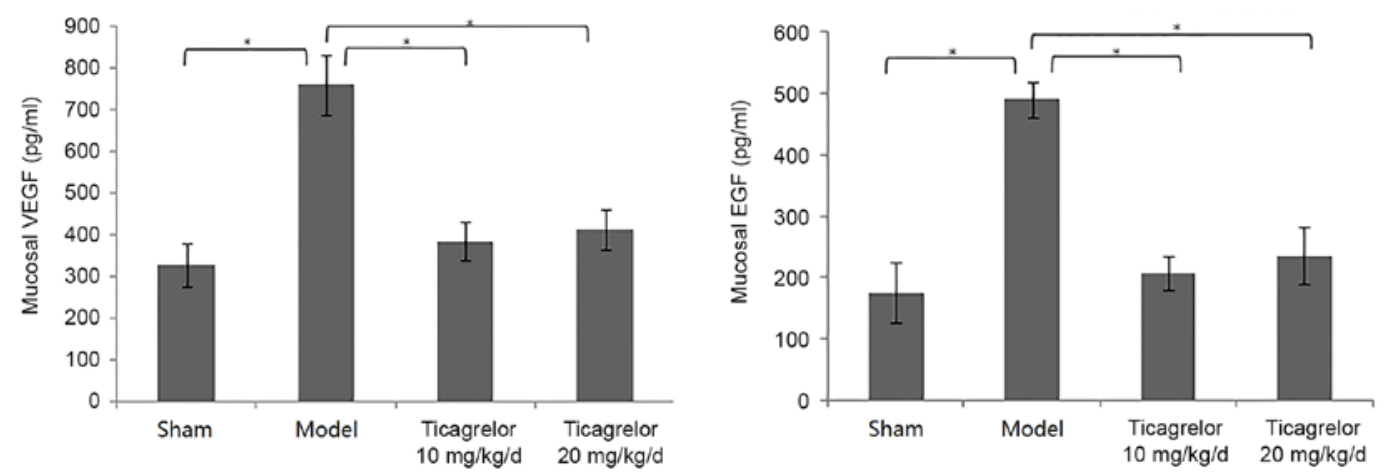

Figure 2. Effects of ticagrelor treatment ( 10 or $20 \mathrm{mg} / \mathrm{kg} / \mathrm{day}$, intragastrically) on the mucosal VEGF and EGF levels at the ulcer margin on day 8 after ulcer induction, as determined by ELISA. Values are presented as the mean \pm standard deviation ( $\mathrm{n}=8$ rats/group). ${ }^{*} \mathrm{P}<0.05$. VEGF, vascular endothelial growth factor; EGF, epidermal growth factor.
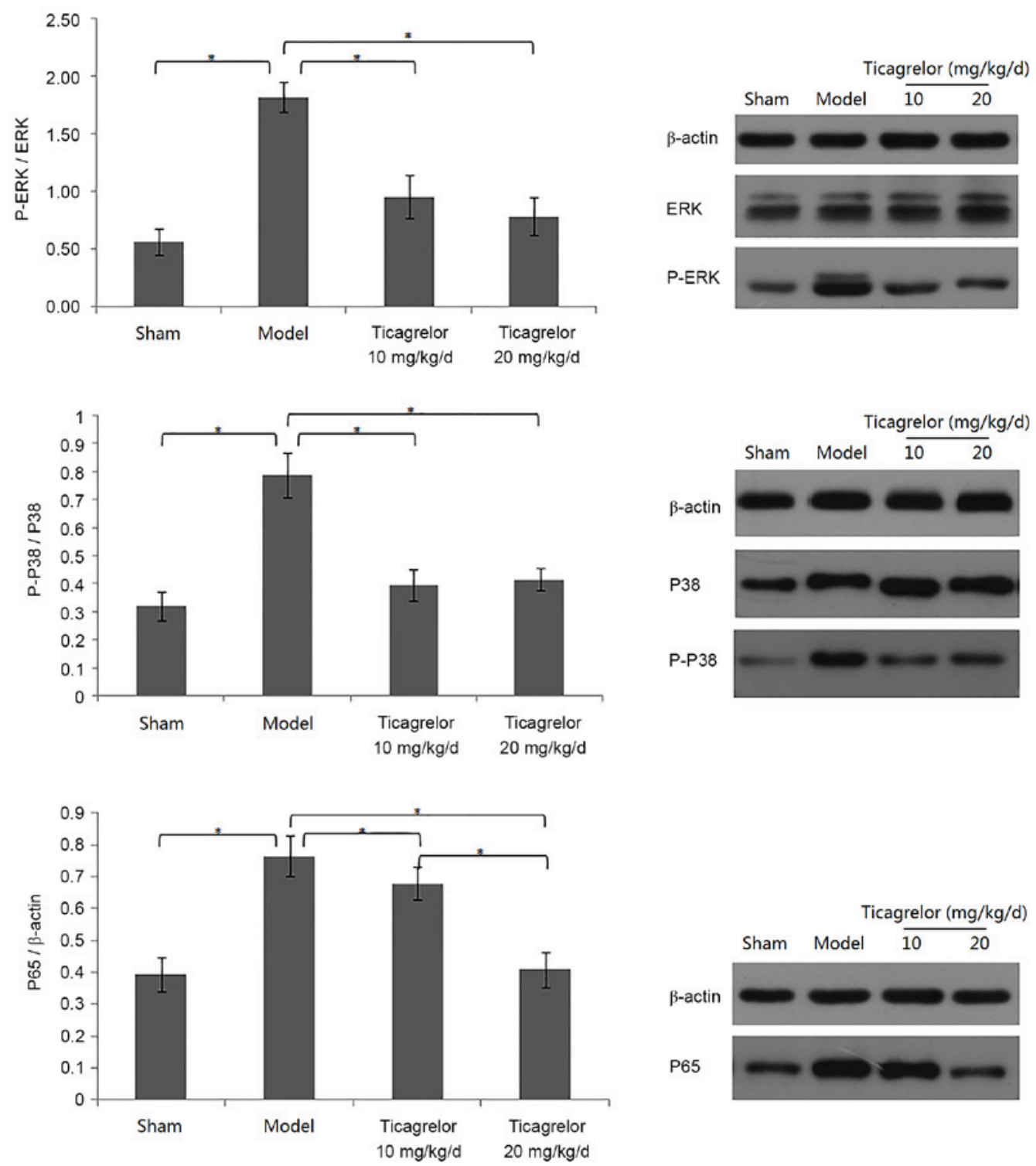

Figure 3. Effects of ticagrelor on the protein expression levels of ERK, P-ERK, P38, P-P38 and nuclear factor-кB P65 at the ulcer margin on day 8 after ulcer induction, as determined by western blot analysis. Values are presented as the mean \pm standard deviation ( $\mathrm{n}=8$ rats/group). ${ }^{*} \mathrm{P}<0.05$. ERK, extracellular signal-regulated kinase; P-, phosphorylated.

and P65 at the ulcer margins when compared with those in sham mucosa $(\mathrm{P}<0.05$; Fig. 3). However, ticagrelor treatment significantly inhibited the ulcer-induced expression levels of P-ERK, P-P38 and P65 at the ulcer margin compared with the 
model group $(\mathrm{P}<0.05$; Fig. 3). And the expression of $\mathrm{P} 65$ in high-dose group was significantly lower than that in low-dose group $(\mathrm{P}<0.05)$.

\section{Discussion}

Accumulated evidence supports the benefits of antiplatelet therapies as a primary and secondary treatment for cardiovascular disease. However, the use of antiplatelet agents has certain risks, in particular, gastrointestinal complications, such as ulceration and resultant bleeding. Previous studies have demonstrated that clopidogrel, a platelet ADP P2 $\mathrm{Y}_{12}$ receptor antagonist, evidently increases gastrointestinal bleeding (6) and delays acetic acid-induced gastric ulcer healing by attenuating gastric epithelial cell proliferation via inhibiting the expression of EGF and the phosphorylation activation of the ERK pathway (11). Ticlopidine, another ADP P2Y 12 receptor antagonist, delays acetic acid-induced gastric ulcer healing and impairs the angiogenesis and healing of rat gastric ulcer by suppressing the release of VEGF (12). However, no previous studies have reported the possible suppression effects of ticagrelor, a relatively novel $\mathrm{P} 2 \mathrm{Y}_{12}$ receptor antagonist, on gastric ulcer healing.

The results of the present study indicated that ticagrelor delayed gastric ulcer healing in a dose-independent manner through reduction of gastric epithelial cell proliferation and angiogenesis. The mean ulcer sizes were larger in the ticagrelor-treated groups when compared with the model group. PCNA is a nuclear protein associated with the cell cycle, whose appearance is correlated with the proliferation state of the cell (11). In the current study, ticagrelor treatment decreased PCNA expression adjacent to the ulcer, which indicated that ticagrelor inhibited cell proliferation. These findings were in line with previous clinical suggestions that ticagrelor is not sufficiently safe for the gastrointestinal mucosa of patients at high risk of developing peptic ulcers (8).

Ulcer healing is a complex process of tissue regeneration, which involves cell proliferation and angiogenesis, ultimately leading to scar formation (13). All these processes are controlled by various growth factors (14). Ulceration triggers the expression of genes encoding for the growth factors, such as EGF and VEGF, in the cells lining the mucosa of the ulcer margin (14). These growth factors produced locally activate epithelial cell migration and proliferation via autocrine and/or paracrine actions. EGF, a polypeptide growth factor, exerts a wide variety of biological effects including the promotion of proliferation, and is essential for gastric ulcer repair and healing (14). Within 3 days after ulcer formation, cells lining the gastric glands in the ulcer margin undergo dedifferentiation, express EGF and its receptor, and actively proliferate (14). EGF in turn locally stimulates cell proliferation, migration and hence ulcer healing (14). Exogenous EGF has been demonstrated to significantly accelerate experimental gastric ulcer healing (15). Angiogenesis is essential for the healing of chronic gastric ulcers, and VEGF is a fundamental regulator of angiogenesis. Following ulceration, cells lining the mucosa of the ulcer margin express VEGF, which then binds to its specific receptors and triggers endothelial cell proliferation, migration and microvascular tube formation and angiogenesis (14). Exogenous VEGF has been observed to markedly accelerate healing of experimental gastric ulcers in rats (16). In the present study, ticagrelor administration reduced EGF and VEGF expression levels, and thus inhibited gastric epithelial cell proliferation and angiogenesis.

MAPKs, including the subgroups of ERK1/2, ERK5, p38 and c-Jun N-terminal kinase, are important signal transducer for cell survival and can regulate several cellular processes including proliferation, differentiation, survival and apoptosis (17). Among them, ERK1/2 mediated EGF-induced cell proliferation and VEGF-induced angiogenesis, while p38 functions as a key component in the induction of cell growth processes, cell differentiation, apoptosis and cellular responses to inflammation. A previous study on experimental gastric ulcers in rats has demonstrated that ulceration triggers increased expression of EGF in epithelial cells of the ulcer margin, and that healing and regeneration of the epithelial component of gastric ulcers involves the activation of the MAPK (ERK1/2) signal transduction pathway (14). Blockage of this pathway with a specific inhibitor of EGF-R kinase clearly delayed gastric ulcer healing (14). The present study revealed that ulcer induction activated the EGF-MAPK (ERK1/2 and P38) signal transduction pathways, while ticagrelor inhibited ulcer-induced the activation of these pathways. Meanwhile, VEGF also has the function of activating the MAPK (ERK1/2) kinase signal transduction pathway that triggers angiogenesis. Previous studies have demonstrated that activation of the MAPK (ERK1/2) kinase signal transduction pathway is crucial for VEGF-induced stimulation of angiogenesis, and that nonsteroidal anti-inflammatory drugs may interfere with angiogenesis by inhibiting MAPK (ERK1/2) pathway (18). The present study revealed that ticagrelor impaired the angiogenesis and healing of rat gastric ulcer by suppressing the release of VEGF via the inhibition of the MAPK (ERK1/2 and P38) signal transduction pathway.

$\mathrm{NF}-\kappa \mathrm{B}$ signaling regulates vascular homeostasis in vivo. Treatment of zebrafish embryos with NF-kB inhibitors provokes vascular leakage and alters vessel morphology (19). Following ulcer induction, thrombi in the submucosal vessels lead to ischemic mucosal necrosis. In the process of ulcer healing, inflammatory cell aggregation, oxidative stress and cell proliferation exacerbate hypoxia in the ulcer. Hypoxia is one of the best characterized stimuli for the induction of VEGF production by a variety of cells and tissues, while VEGF expression is upregulated by hypoxia-induced mitogenic factor through activation of the NF- $\mathrm{kB}$ pathway (20). In the present study, ulcer induction markedly induced phosphorylation of NF- $\mathrm{BB}$ P65, whereas administration of ticagrelor impaired phosphorylation of these molecules.

The low-dose of ticagrelor used in the present study $(10 \mathrm{mg} / \mathrm{kg} / \mathrm{day}$ in rats) is similar to the dose used in previous studies (10) and in clinical practice $(90 \mathrm{mg}$ twice a day for humans) (7). Furthermore, $20 \mathrm{mg} / \mathrm{kg} / \mathrm{day}$ was applied as the high-dose in the present study. Therefore, the inhibitory effect of ticagrelor on gastric ulcer healing in the current study may resemble a real-life situation.

In conclusion, the present study revealed that ticagrelor administration delayed gastric ulcer healing in rats in a dose-independent manner. The possible underlying mechanisms were associated with the functions of ticagrelor in attenuating the expression levels of VEGF and EGF, as well as 
suppressing the phosphorylation activation of ERK1/2,p38 and $\mathrm{NF}-\kappa \mathrm{B}$ P65. Finally, the gastric epithelial cell proliferation and angiogenesis were inhibited following ticagrelor treatment.

\section{Acknowledgements}

This study was supported by the Natural Science Fund of Health and Family Planning Commission of Wuhan Municipality, China (grant nos. WX17D12 and WX12C32).

\section{References}

1. Amsterdam EA, Wenger NK, Brindis RG, Casey DE Jr, Ganiats TG, Holmes DR Jr, Jaffe AS, Jneid H, Kelly RF, Kontos MC, et al: 2014 AHA/ACC guideline for the management of patients with non-ST-elevation acute coronary syndromes: A report of the American college of cardiology/American heart association task force on practice guidelines. Circulation 130: e344-e426, 2014.

2. Roffi M, Patrono C, Collet JP, Mueller C, Valgimigli M, Andreotti F, Bax JJ, Borger MA, Brotons C, Chew DP, et al: 2015 ESC Guidelines for the management of acute coronary syndromes in patients presenting without persistent ST-segment elevation: Task force for the management of acute coronary syndromes in patients presenting without persistent ST-Segment elevation of the European society of cardiology (ESC). Eur Heart J 37: 267-315, 2016.

3. Serebruany VL, Dinicolantonio JJ, Can MM, Pershukov IV and Kuliczkowski W: Gastrointestinal adverse events after dual antiplatelet therapy: Clopidogrel is safer than ticagrelor, but prasugrel data are lacking or inconclusive. Cardiology 126 35-40, 2013.

4. McQuaid KR and Lalne L: Systematic review and meta-analysis of adverse events of low-dose aspirin and clopidogrei in randomized controlled trials. Am J Med 119: 624-638, 2006.

5. Di Minno A, Spadarella G, Prisco D, Scalera A, Ricciardi E and Di Minno G: Antithrombotic drugs, patient characteristics, and gastrointestinal bleeding: Clinical translation and areas of research. Blood Rev 29: 335-343, 2015.

6. Bhatt DL, Scheiman J, Abraham NS, Antman EM, Chan FK, Furberg CD, Johnson DA, Mahaffey KW, Quigley EM, Harrington RA, et al: ACCF/ACG/AHA 2008 expert consensus document on reducing the gastrointestinal risks of antiplatelet therapy and NSAID use: A report of the American college of cardiology foundation task force on clinical expert consensus documents. J Am Coll Cardiol 52: 1502-1517, 2008.

7. Wallentin L, Becker RC, Budaj A, Cannon CP, Emanuelsson H, Held C, Horrow J, Husted S, James S, Katus H, et al: Ticagrelor versus clopidogrel in patients with acute coronary syndromes. $\mathrm{N}$ Engl J Med 361: 1045-1057, 2009.
8. DiNicolantonio JJ, D'Ascenzo F, Tomek A, Chatterjee S, Niazi AK and Biondi-Zoccai G: Clopidogrel is safer than ticagrelor in regard to bleeds: A closer look at the PLATO trial. Int J Cardiol 168: 1739-1744, 2013

9. Tsukimi Y and Okabe S: Validity of kissing gastric ulcers induced in rats for screening of antiulcer drugs. J Gastroenterol Hepatol 9 (Suppl 1): S60-S65, 1994.

10. Grzesk G, Kozinski M, Navarese EP, Krzyzanowski M, Grzesk E, Kubica A, Siller-Matula JM, Castriota F and Kubica J: Ticagrelor, but not clopidogrel and prasugrel, prevents ADP-induced vascular smooth muscle cell contraction: A placebo-controlled study in rats. Thromb Res 130: 65-69, 2012.

11. Luo JC, Huo TI, Hou MC, Lin HY, Li CP, Lin HC, Chang FY and Lee FY: Clopidogrel delays gastric ulcer healing in rats. Eur J Pharmacol 695: 112-119, 2012.

12. Ma L, Elliott SN, Cirino G, Buret A, Ignarro LJ and Wallace JL: Platelets modulate gastric ulcer healing: Role of endostatin and vascular endothelial growth factor release. Proc Natl Acad Sci USA 98: 6470-6475, 2001.

13. Khoder G, Al-Menhali AA, Al-Yassir F and Karam SM: Potential role of probiotics in the management of gastric ulcer. Exp Ther Med 12: 3-17, 2016.

14. Tarnawski AS and Ahluwalia A: Molecular mechanisms of epithelial regeneration and neovascularization during healing of gastric and esophageal ulcers. Curr Med Chem 19: 16-27, 2012.

15. Brzozowski T, Konturek PC, Konturek SJ, Schuppan D, Drozdowicz D, Kwiecień S, Majka J, Nakamura T and Hahn E: Effect of local application of growth factors on gastric ulcer healing and mucosal expression of cyclooxygenase-1 and -2 . Digestion 64: 15-29, 2001.

16. Jones MK, Kawanaka H, Baatar D, Szabó IL, Tsugawa K, Pai R, Koh GY, Kim I, Sarfeh IJ and Tarnawski AS: Gene therapy for gastric ulcers with single local injection of naked DNA encoding VEGF and angiopoietin-1. Gastroenterology 121: 1040-1047, 2001.

17. Plotnikov A, Zehorai E, Procaccia S and Seger R: The MAPK cascades: Signaling components, nuclear roles and mechanisms of nuclear translocation. Biochim Biophys Acta 1813: 1619-1633, 2011.

18. Tarnawski AS and Jones MK: Inhibition of angiogenesis by NSAIDs: Molecular mechanisms and clinical implications. J Mol Med (Berl) 81: 627-636, 2003.

19. Santoro MM, Samuel T, Mitchell T, Reed JC and Stainier DY: Birc2 (clap1) regulates endothelial cell integrity and blood vessel homeostasis. Nat Genet 39: 1397-1402, 2007.

20. Tabruyn SP and Griffioen AW: NF-kappa B: A new player in angiostatic therapy. Angiogenesis 11: 101-106, 2008. 